\title{
Paperlike thermochromic display
}

\author{
Liyu Liu, Suili Peng, Weijia Wen, ${ }^{\text {a) }}$ and Ping Sheng \\ Department of Physics, The Hong Kong University of Science and Technology, Clear Water Bay, Kowloon, \\ Hong Kong and Institute of Nano Science and Technology, The Hong Kong University of Science \\ and Technology, Clear Water Bay, Kowloon, Hong Kong
}

(Received 2 April 2007; accepted 30 April 2007; published online 23 May 2007)

\begin{abstract}
The authors report the design and implementation of a paperlike, thermally activated display fabricated from thermochromic composite and embedded conductive wiring patterns, shaped from mixture of metallic nanoparticles in polydimethylsioxane using soft lithography. The display exhibits good image quality and ease of control. Use of electric heating pulses is shown to reduce energy consumption while improving image quality control. The display has excellent mechanical bending flexibility. (C) 2007 American Institute of Physics. [DOI: 10.1063/1.2742781]
\end{abstract}

Flexible display devices offer the advantages of lightweight and portability. ${ }^{1,2}$ Many flexible display devices are based on liquid crystals combined with polymeric structures. For example, displays with high flexibility can be fabricated using liquid crystal encapsulated as single pixels in elastomer substrate, ${ }^{3}$ or in field-induced polymer structures. ${ }^{4}$ To drive the displays, conducting wires/patterns are indispensable for transmitting the controlling signals. Recently, an ultralow-power organic circuit has been realized. ${ }^{5}$ It was reported that the electric circuits can be fabricated with electric and photolithography, ${ }^{6,7}$ direct ink-jet printing with conductive compositions, ${ }^{8,9}$ etc.

Here we report the design and fabrication of thermally activated display using films made of thermochromic composite and embedded conductive wiring patterns. Thermochromic powder is a material whose optical properties (e.g., color) are tunable by varying the temperature, in a reversible and repeatable manner. Preparations of such material have been mainly studied with respect to the reversible thermochromic effect. ${ }^{10-12}$ Owing to the accurate, rapid, and stable characteristics, ${ }^{13}$ this material promises broad applications ranging from smart windows, color filters, and temperature sensors. ${ }^{14,15}$ Polydimethylsioxane (PDMS) plays an important role for our thermal displays, mainly due to its desirable wetting characters with thermochromic nanoparticles and silver powders. Thus the thermochromic or conducting polymer gel can be easily made. ${ }^{16}$

The display is based on two composite materials: thermochromic polymers and silver-PDMS conductive composites. Microencapsulated thermochromic powder $(3-7 \mu \mathrm{m}$ in diameter, Lijinkeji Co. Ltd) is employed whose color is dark green at room temperature and turns white above $60{ }^{\circ} \mathrm{C}$. When the powder is mixed with PDMS 2025 (Dow Coning 184 ) at a particle concentration of $20 \%(\mathrm{w} / \mathrm{w})$ and thoroughly grinded, a liquidlike composite is formed that has a darkgreen color. To prepare the conducting composite, micronsized silver powder $(1.2-2.2 \mu \mathrm{m})$ is used and mixed with PDMS at the silver concentration of $86.3 \%$ (w/w). After vigorously stirred, the composite forms a gel-like soft mixture. With soft-lithographic technique, the conducting composite offers advantages of ease in patterning microconducting wires and in integrating electrical circuits. When the thermo-

\footnotetext{
${ }^{\text {a) }}$ Author to whom correspondence should be addressed; electronic mail:
} phwen@ust.hk chromic composite is spinned at a speed of $400 \mathrm{rpm}$ for $18 \mathrm{~s}$ onto the designed patterns and cured after a short bake, a thermochromic display is formed, with the thickness of $\sim 150 \mu \mathrm{m}$. Owing to the PDMS matrix, the thermochromic and conducting composite exhibits polymeric properties with excellent flexibility. The ease in shaping the conducting patterns offers a great advantage in the design of our display devices.

Figure 1 is a three-dimensional picture showing the structure of the display cell. It is basically a single layer of thermochromic sheet in which the conductive wire pattern, in the shape of a logo, is embedded. When a voltage is applied to the two outstretched electrodes, the resulting electrical current will generate localized heating to the thermochromic layer that lies directly above the conducting wires. ${ }^{17}$ Once the local temperature rises to $60{ }^{\circ} \mathrm{C}$ or above, the color of the thermochromic layer promptly turn from dark green to white, leading to a visible, white image of the logo. As the average thermal diffusivity of the thermochromic composite is very small, e.g, about $2.4 \times 10^{-3} \mathrm{~cm}^{2} \mathrm{~s}^{-1}$, the logo will remain sharp with well controlled localized temperatures and will not be blurred via thermal conduction. For accurate and ease of control, the conducting pattern has been designed as a series circuit to ensure that the same current passes through the whole path. Local conductance of the pattern is deter-

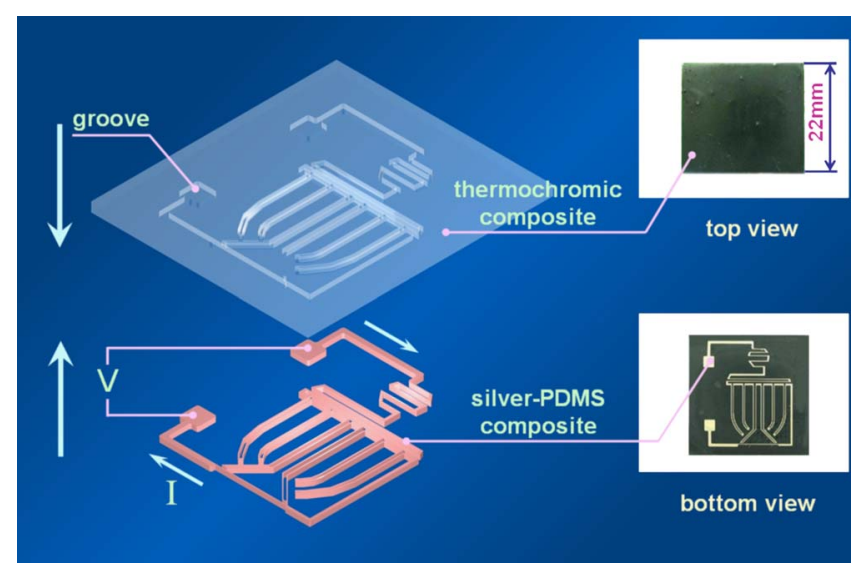

FIG. 1. (Color online) Schematic illustrations of the display structure. The logo-patterned conductive wirings are shaped from silver microparticlePDMS composite by using soft lithography. The conductive wire pattern is embedded into the thermochromic sheet. Insets at the right show the top and bottom views of the fabricated device. 


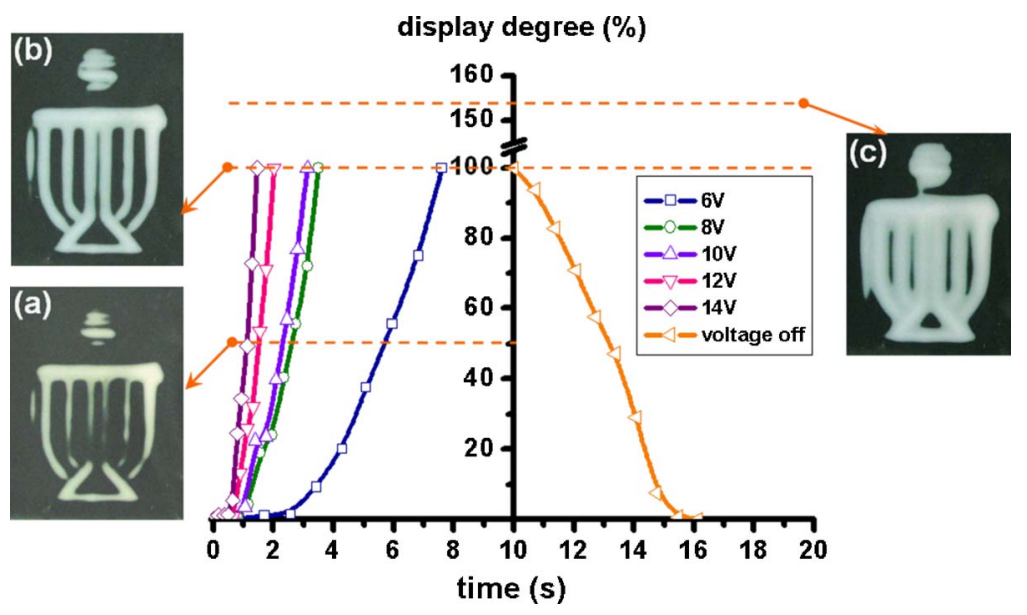

FIG. 2. (Color online) Display degree plotted as a function of applied voltage. The five curves on the left correspond to a step-function voltage of various height, while the one on the right corresponds to what happens after the voltage is turned off. The insets show the logo images at various display degrees. The image in inset (c) is blurred due to overheating.

mined by the width of the conducting lines: lines for generating heat need higher resistance and therefore are designed to have a $100 \mu \mathrm{m}$ width; others for electrical conduction are wider $(300 \mu \mathrm{m})$ so as to lower the resistance. The upper right inset of Fig. 1 is a top view of the display which is a 22-mm-wide square. The thermochromic material completely covers the conducting patterns and presents a uniform dark-green colors. The conducting wires are visible in the bottom view, shown in the lower right inset.

An important feature in the performance of a display is the response time to the applied voltage. The experiment was carried out at an ambient temperature of $20.4{ }^{\circ} \mathrm{C}$, on a testing sample with $80 \Omega$ resistance. A charge coupled device camera is employed to record image evolution when the thin thermochromic film is subjected to a step-function dc voltage. Images were arranged in a time sequence, and the image which yields the most complete and accurate logo has been found, which is shown as an inset (b) in Fig. 2. This image was then defined as $100 \%$ display degree (clearest). Here images' display degrees are determined by using a commercial software (PHOTOSHOP). The images' display degree versus time under different voltages are plotted in Fig. 2. In two cases, shown in insets (a) and (c), the corresponding images are also displayed. They are clearly inferior to that shown in inset (b). The pictures on the left side of Fig. 2 illustrate the speed at which the images appear after the voltage has been applied. It is seen that as the voltage is increased from 6 to $14 \mathrm{~V}$, the response time is significantly decreased. At a fixed voltage, the display degree increases with time. When the voltage is higher than $8 \mathrm{~V}$, the film is capable of attaining a clear image within $2 \mathrm{~s}$. We found that by increasing either the time duration or voltage of the applied voltage, the display can be overheated, leading to a blurred image, as shown in inset (c) on the right side of Fig. 2.

To overcome the problem of overheating, periodic square pulse trains with a fixed duty cycle is used. This can avoid excessive heating, maintain the desired clear image, as well as decrease power consumption. To optimize the square pulse duration $t$ and voltage $V$, a series of experiment was carried out with the pulse period $T$ fixed at $20 \mathrm{~ms}$. The table in Fig. 3 gives the best values of $V$ (for best images) under different $t / T$ ratios, ranging from $5 \%$ to $50 \%$. It is evident that with decreasing $t / T$, the best $V$ value increases monotonically. Power consumption as a function of $t / T$ can also be calculated. As the resistivity of the silver-PDMS conducting composite can increase $70 \%$ from $22^{\circ} \mathrm{C}$ to $60^{\circ} \mathrm{C}$, ${ }^{18}$ the Downloaded 28 Jun 2007 to 143.89 .104 .110 . Redistribution subject resistance of the conducting pattern would be $136 \Omega$ in the display mode. Power consumption $W$ is thus given by $W$ $=\left(V^{2} \times(t / T)\right) / R$, which is plotted in Fig. 3 as the solid line, where the $V$ value used is that for the best images. The solid squares are the measured values. Good agreement is seen. It shows that the energy can be reduced to a minimum of $0.13 w$ at $t / T \sim 40 \%$. When the $t / T$ value surpasses the optimal point, the power consumption increases rapidly. Thus the minimum in the power consumption is the result of the competition between pulse duration and the applied voltage. While a decrease in $t / T$ favors energy reduction, the coincident increase in the best $V$ value would offset this reduction through the $V^{2}$ dependence. Based on the above discussion, the application of periodic square pulse train not only solves the problem of overheating but can also lower power consumption.

The mechanical property of the PDMS-based thermochromic material and conducting composite endows the thermochromic display with high flexibility. The thickness of the film, $\sim 150 \mu \mathrm{m}$, enables the film to bend, fold, and distorted at discretion while preserving the normal displaying functions. Figure 4(a) shows the display to be wrapped around a column. Once a voltage is applied, the logo image appears promptly, as shown in Fig. 4(b). With such mechanical flexibility, our thin-film thermochromic display can easily adapt to a variety of application environments.

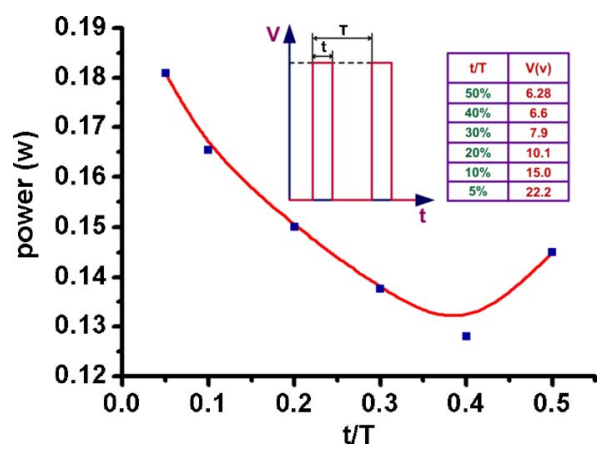

FIG. 3. (Color online) Power consumption of the display under different $t / T$ ratios of the heating pulse train. The duty cycle is fixed at $50 \mathrm{~Hz}$. The table gives the best voltage values (for achieving an accurate image) associated with the various values of $t / T$ ratio. Solid curve is calculated from the expression given in the text. Solid squares are measured data.

to AIP license or copyright, see http://apl.aip.org/apl/copyright.jsp 


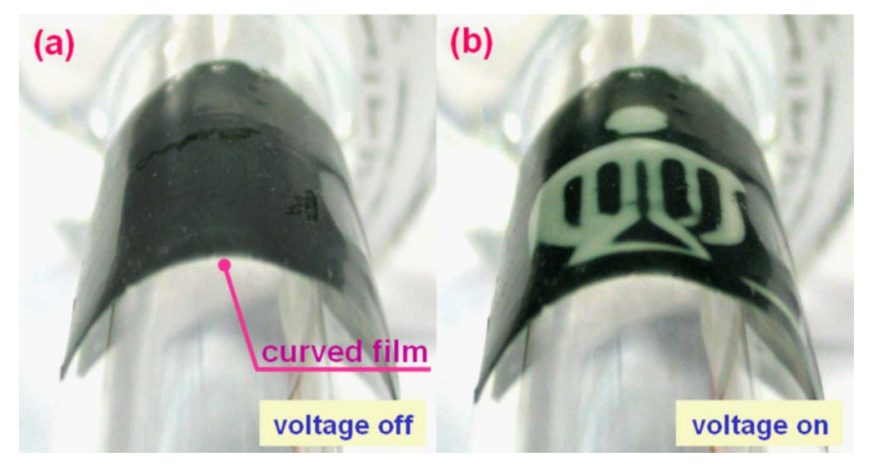

FIG. 4. (Color online) Display's function is shown to be not affected by mechanical distortion. Here, the display is wrapped on a column. (a) Shows the display film when no input signal is applied, and (b) shows the logo image to be correctly displayed when a voltage heating pulse train is applied.

Based on the ease of fabrication and simple architecture, the thermochromic display can have advantages in lowering the display unit cost. The heating pulse control scheme can also lower power consumption, and the lightweight and mechanical flexibility can mean portability and convenience. With matrixlike thermal pixels, programmable images can be generated with digital control.

The authors acknowledge the support from Hong Kong RGC under Grant No. HKUST 621006.
${ }^{1}$ R. A. Street, W. S. Wong, S. E. Ready, M. L. Chabinyc, A. C. Arias, S. Limb, A. Salleo, and R. Lujan, Mater. Today 9, 32 (2006).

${ }^{2}$ L. Zhou, A. Wanga, S. C. Wu, J. Sun, S. Park, and T. N. Jackson, Appl. Phys. Lett. 88, 083502 (2006).

${ }^{3}$ Y. T. Kim, J. H. Hong, T. Y. Yoon, and S. D. Lee, Appl. Phys. Lett. 88, 263501 (2006)

${ }^{4}$ E. A. Büyüktanir, N. Gheorghiu, J. L. West, M. Mitrokhin, B. Holter, and A. Glushchenko, Appl. Phys. Lett. 89, 031101 (2006).

${ }^{5}$ C. Balocco, L. A. Majewski, and A. M. Song, Org. Electron. 7, 500 (2006).

${ }^{6}$ H. Klauk, U. Zschieschang, J. Pflaum, and M. Halik, Nature (London) 445, 745 (2007).

${ }^{7}$ W. Shen, Y. Chen, and Q. Pei, Appl. Phys. Lett. 87, 124106 (2005).

${ }^{8}$ D. Kim, S. Jeong, B. K. Park, and J. Moon, Appl. Phys. Lett. 89, 264101 (2006).

${ }^{9}$ J. M. Leger, A. L. Holt, and S. A. Carter, Appl. Phys. Lett. 88, 111901 (2006).

${ }^{10}$ Y. Noguchi, T. Sekitani, and T. Someya, Appl. Phys. Lett. 89, 253507 (2006).

${ }^{11}$ M. Seredyuk, A. B. Gaspar, V. Ksenofontov, S. Reiman, Y. Galyametdinov, W. Haase, E. Rentschler, and P. Gütlich, Chem. Mater. 18, 2513 (2006).

${ }^{12}$ A. Seeboth, J. Kriwanek, and R. Vetter, Adv. Mater. (Weinheim, Ger.) 12, 1424 (2000).

${ }^{13}$ C. R. Smith, D. R. Sabatino, and T. J. Praisner, Exp. Fluids 30130 (2001).

${ }^{14}$ A. Mills, and A. Lepre, Analyst (Cambridge, U.K.) 124, 685 (1999).

${ }^{15}$ M. G. Baron and M. Elie, Sens. Actuators B 90271 (2003).

${ }^{16}$ Y. N. Xia and G. M. Whitesides, Annu. Rev. Mater. Sci. 28, 153 (1998).

${ }^{17}$ L. Liu, S. Peng, X. Niu, and W. Wen, Appl. Phys. Lett. 89, 223521 (2006).

${ }^{18}$ X. Niu, S. Peng, L. Liu, W. Wen, and P. Sheng, Adv. Mater. (Weinheim, Ger.) (in press). 